\title{
Nonlinear Characterization of Rhodamine 610 dye-doped PMMA Thin Films Under 650 nm CW Laser Light Excitation
}

\author{
Amal F. Jaffar \\ Optometry Department, Institute of Medical Technology, Al-Mansour, Foundation of Technical \\ Education, Ministry of High Education \& Scientific Research. \\ E-Mail: amaljaffar@yahoo.com.
}

\begin{abstract}
In this work the nonlinear optical properties for Rodamine 610 (R610) in PMMA doped films with different concentrations with the solvent Chloroform by using a high sensitive method known as Z-Scan technique were studied.

Z-scan experiment was performed using CW diode laser at $650 \mathrm{~nm}$ with the power output of $45 \mathrm{~mW}$, in two parts. The first part was done using a closed-aperture placed in front of the detector to measure the nonlinear refractive index. In the second part; the aperture in front the detector was removed (open aperture) to measure the nonlinear absorption coefficient. The experimental results for closed aperture show that the dye doped films exhibit negative refractive index (self_defocusing) for the films of concentration $10^{-5}$ and $10^{-4} \mathrm{M}$, while the film of concentration $10^{-6} \mathrm{M}$ exhibits positive refractive index (self-focusing), The results show also that the nonlinear refractive index and the third order nonlinear susceptibility increased with the increasing of the concentration due to the increasing of the film thickness which caused increasing the nonlinear phase shift in the samples. With open aperture z-scan the samples exhibit two photon absorption under the experimental conditions. The effective values of the nonlinear refractive index $\mathrm{n}_{2}$, the nonlinear absorption coefficient $\beta$, real and imaginary parts of the third-order optical nonlinearity, $\chi^{(3)}$ were evaluated. The results imply that R610 films can be used as a potential medium for various optoelectronic applications including that in optical power limiting.
\end{abstract}

Keywords: Nonlinear optics, z-scan technique, Dye doped polymer films.

\section{Introduction}

Rapid technological advancements in optics have placed great demand on the development of nonlinear optical (NLO) materials with prominent applications in optical limiting and all optical switching. Extremely large number of organic compounds with delocalized electron and conjugated double bond systems and a large dipole moment have been synthesized to realize the susceptibilities far larger than the inorganic optical materials [1].

Dye chromophores are a class of organic molecules with multiple $\pi$-conjugated bonds, which can exhibit large optical nonlinearities and fast response time, as a result of the ease of polarization of their extended mobile $\pi$-electron clouds over large molecular distances. Strong absorption of dyes in the visible region makes them particularly suited for nonlinear optical investigations .It has also been shown that embedding dye chromophores in suitable host matrices enhances the lifetime and stability of the dyes entrapped within it
[2]. Dye doped polymers find applications in the fields of modern photonic technology apart from its use as an alternative to solid state laser media. [3].

$Z$-scan technique based on the spatial distortion of a laser beam passed through a NLO material is widely used in material characterization because of its simplicity, high sensitivity and wellelaborated theory. The opportunity to conduct simultaneous measurements of various NLO parameters in one set of experiments also makes this technique attractive and applicable for different materials. This different materials .This method yields both the sign and the magnitude of the nonlinearity, and the value of the nonlinear refractive index $n_{2}$ may be easily extracted from experimental data with a minimum of analysis $[4,5]$.

There were two parts of the Z-scan: closed aperture and open aperture. Closed aperture Z-scan helps to measure the sign and magnitude of both real \& imaginary part of third order (NLO) and nonlinear refractive index $\left(\mathrm{n}_{2}\right)$.Open aperture Z-scan either two types: saturable absorption (SA) and reverse 
saturable absorption (RSA). Depending on the pump intensity and on the absorption cross section at the excitation wavelength. Open aperture Z-scan helps to measure the nonlinear absorption coefficient $B_{2}$ [6].

In this paper, the optical nonlinearity of dye thin films doped with PMMA at $50 \mathrm{~mW} \mathrm{cw}$ diode laser power at wavelength of $650 \mathrm{~nm}$ was studied. The experiment was repeated for different dye concentrations, the third order nonlinear refractive index and the nonlinear absorption coefficient were found to be linearly dependent on the dye concentration within the range studied.

\section{Experimental Section:}

$\mathrm{R} 610$ is from Xanthene family with fluorescence emission in the yellow -red region of the electromagnetic spectrum are well known for their excellent laser performance in liquid solutions as well as in solid matrices [6]. Fig. (1) shows its molecular structure.

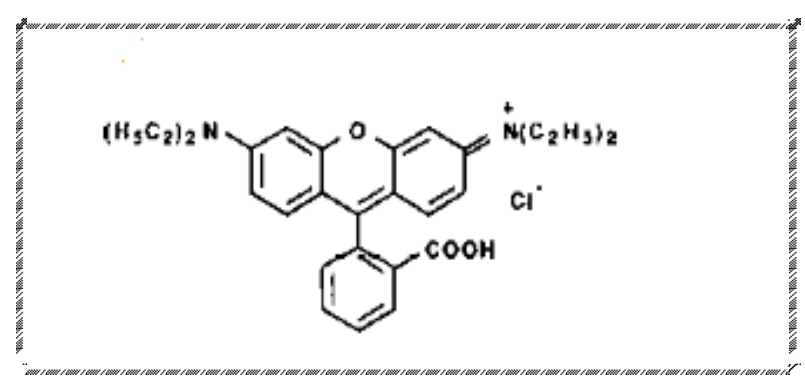

Fig. (1) The molecular structure of $R$ 610, [7].

Polymethylmethacrylate (PMMA) is from ICI Company. The spectroscopic grade solvent Chloroform is from Lab-Scan LTd., Analitical Science HPLC Ireland-Dublin.

\section{Samples Preperation}

Solution of concentrations $\left(10^{-6}, 10^{-5}\right.$ and $\left.10^{-4}\right) \mathrm{M}$ in Chloroform where prepared by weighting amount of the material by using a matter balance having a sensitivity of $10^{-4} \mathrm{gm}$. To enhance the solubility of the dye in the solvent, the dye firstly dissolved in Methanol with ratio $(20 \%)$.

Dye doped polymer films were fabricated by the free casting technique_(FC). Polymer solution was prepared by dissolving the required amount of polymer $(7 \mathrm{gm}$. in $100 \mathrm{ml}$ of the used solvent).
The free casting (FC), involves casting a polymer solution in a flat bottomed glass cup (Petri dish) without imposing hydrodynamic stress on the liquid .The dishes were arranged on a glass plate in order the dishes have a plan situation, then the dishes were covered with a heavy paper box to be protect from light and dust. .Solvent is allowed to evaporate under ambient conditions $\left(30 \mathrm{c}^{\mathrm{o}}\right)$ until the films hardens. The hardend films were then removed from the Petri dish by washing it off with distilled water and placed in the oven $\left(50 \mathrm{c}^{\mathrm{o}}\right)$ for 10 minutes to dry .The thickness of the films produced by this method is dependent in straight forward way on concentration and the volume of the liquid in the dish. In this study the liquid volume was (10) $\mathrm{ml}$, yielding film thicknesses in the range (171_191) $\mu \mathrm{m}$ for different cocentrations. The thicknesses of the films were measured with an electrical device (Mini-test 3000 microprocessor coating thickness) from electro, phyisk, Germany (ERICHSEN).

UV-Visible absorption spectra of the lasing dye were carried out by using UV-Visible Shimadzu spectrophotometer. (UV 160), which operates in wavelength range of $200 \mathrm{~nm}$ to $1100 \mathrm{~nm}$ and with scanning speed of $1500(\mathrm{~nm} / \mathrm{min})$, carried out UV-Visible spectroscopy measurements.

The nonlinear optical properties were investigated employing the standard Z-scan technique schematically shown in Fig.(2), and the photographic picture shown in Fig.(3).

The $Z$-scan experiments were performed using a $650 \mathrm{~nm} \mathrm{CW}$ diode laser, max.power is $50 \mathrm{~mW}$, beam diameter: $1.5 \mathrm{~mm}$, beam divergent $1.5 \mathrm{mrad}$, which was focused by $10 \mathrm{~cm}$ focal length lens. The laser beam waist $\omega_{0}$ at the focus is measured to be $0.015 \mathrm{~mm}$ and the Rayleigh length to be $0.0138 \mathrm{~cm}$. 


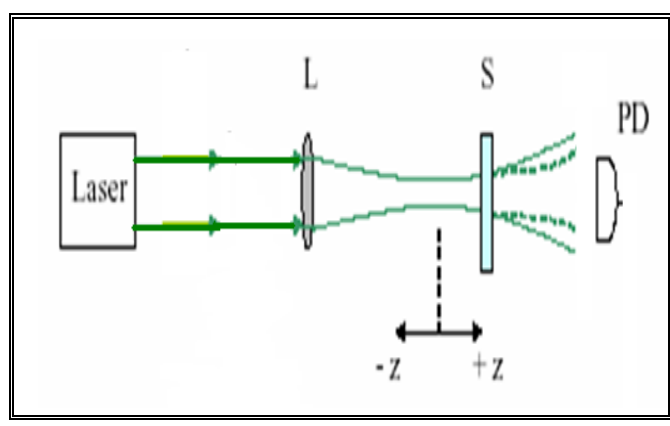

(a)

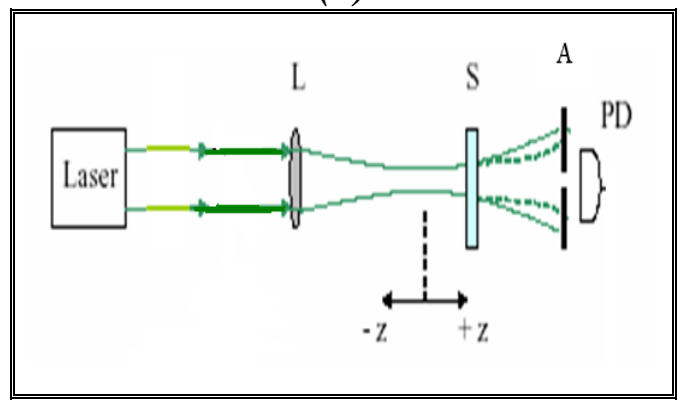

(b)

Fig. (2) (a) Open Aperture Z-Scan, (b) Closed Aperture Z-scan. [8].

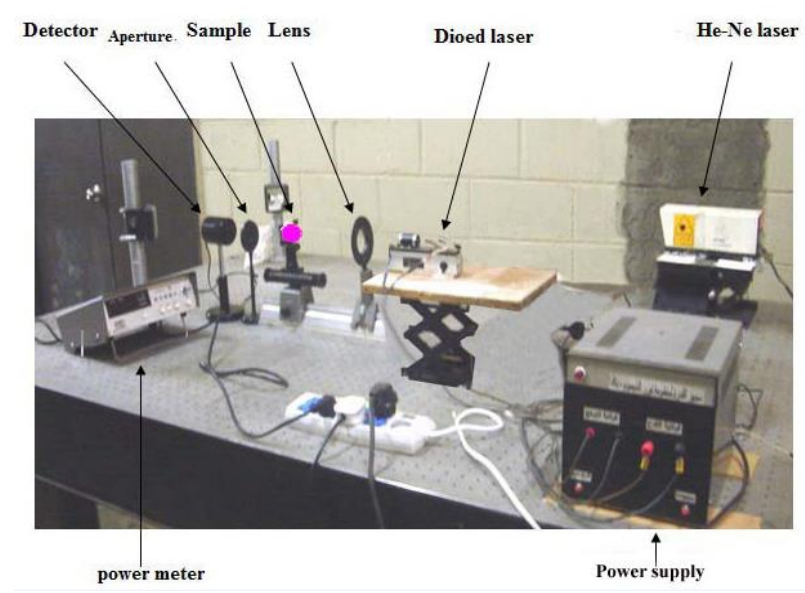

Fig. (3) Photographic picture for the experiment setup.

For this, the transmittance through the sample is monitored as a function of the incident laser light intensity, with and without an aperture in the far field, while the sample is gradually translated along the optical axis of a convex lens. The intensity dependent transmission through the sample measured as a function of sample position with respect to the focal plane, without an aperture (open aperture (OA), Fig.(2a)) gives information about purely absorptive nonlinearity; whereas the transmission measured through an aperture inserted in the far field (closed aperture (CA), Fig.(2b) includes the effect of both nonlinear refraction and nonlinear absorption, if any, the division of the normalized closed aperture Z-scan data by the open aperture Z-scan data generates a Z-scan profile pertaining to the purely dispersive (refractive) nonlinearity [9]. Z-scan method provides a direct measurement of the real and imaginary parts of the thirdorder nonlinear optical susceptibility $\chi^{(3) \text {. }}$.

The sign of the nonlinearity is an important parameter for practical realization of optical signal processing devices [10]. This information cannot be obtained from any other techniques such as degenerate four waves mixing or third harmonic generation. In general, the manifestation of strong nonlinear absorption enhances the optical limiting properties, whereas that of strong nonlinear refraction facilitates the switching properties of organic molecules [11].

Closed aperture Z-scan technique is based on the transformation of phase distortions to amplitude distortions during beam propagation. A qualitative physical argument that explains the transmittance variations in the Z-scan experiment can be given as follows: Starting the scan from a distance far away from the focus (negative $\mathrm{z}$ ), the beam irradiance is low and negligible nonlinear refraction occurs leading to linear transmittance. As the sample is brought closer to the focus, the beam irradiance increases leading to self-lensing in the sample. A negative self-lensing (self-defocusing) prior to focus tends to collimate the beam and reduce the diffraction leading to a smaller beam at the aperture and an increased transmittance. As the scan continues and the sample crosses the focal plane to the right (positive $\mathrm{z}$ ), the same self-defocusing effect will tend to augment diffraction and reduce the aperture transmittance. A prefocal transmittance maximum (peak) and a post focal transmittance minimum (valley) will be, the $\mathrm{z}$-scan signature of a negative nonlinearity as shown by the dote line Fig.(3), while a positive one, following the same analogy, will give rise to an opposite valley-peak configuration, as shown by Solid line Fig. (4). [9]. 


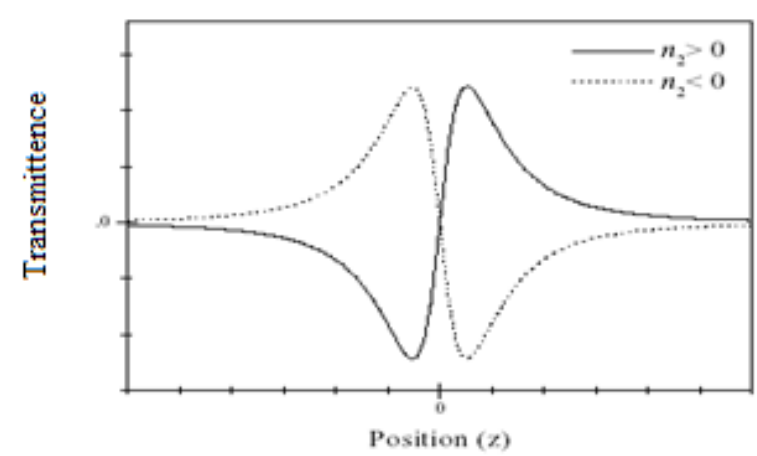

Fig.(4) Calculated Z-scan transmittance curves for a third order nonlinearity [8].

\section{Self Focusing and Defocusing}

Self-focusing (or Kerr-lansing) is a consequence of the non-uniform spatial profile of the laser Gaussian beam. If the intensity of a transmitted light beam is sufficiently high, the refractive index change will modify the light propagation not only with respect to the polarization but in its geometrical properties too [12].

For a Gaussian beam of radius $\omega_{\mathrm{o}}$ (beam waist) the Kerr-lens focal length is:

$\mathrm{F}=\mathrm{a} \omega_{\mathrm{o}}^{2} / 4 \mathrm{tn}_{2} \mathrm{I}$

Where $t$ is the thickness of the nonlinear medium (sample), $\mathrm{I}$ is the irradiance and $\mathrm{a}$ is a correction term.

When $n_{2}$ is negative, the above equation shows there will be a negative focal length and thus self de-focusing of the incident beam [8].

\section{Results and Discussion}

The absorption spectra of dye doped polymer films for various dye concentrations $10^{-6} \mathrm{M}, 10^{-5} \mathrm{M}$, and $10^{-4} \mathrm{M}$ are illustrated in Fig.(5). The spectra of the dye molecule exhibit a wide absorption band with distinct peaks, which are located at $560 \mathrm{~nm}$.

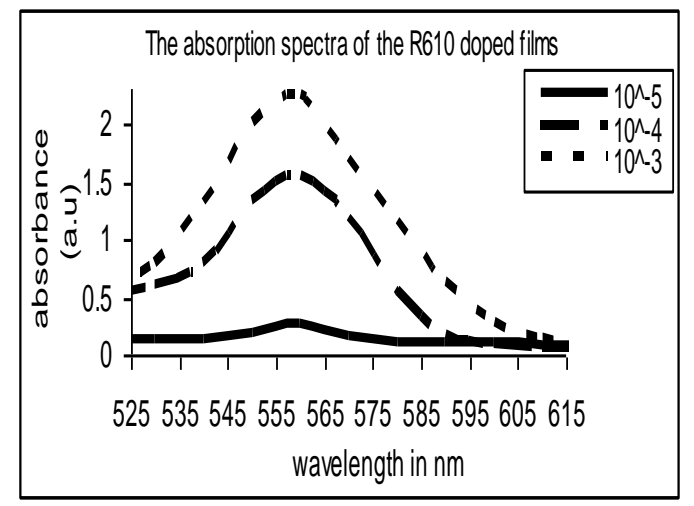

Fig.(5) Linear absorption spectra of R610 Doped films.
The third-order nonlinear refractive index $\mathrm{n}_{2}$ and the nonlinear absorption coefficient $\beta$, of the R610 dye doped polymer films in PMMA at various concentrations for the incident intensity $\mathrm{I}_{0}=509.6 \mathrm{~W} / \mathrm{cm}^{2}$ were evaluated by the measurements of Z-scan. Fig.(6) shows the typical OA Z-scan profiles of the solid films for the three different concentrations of the dye.

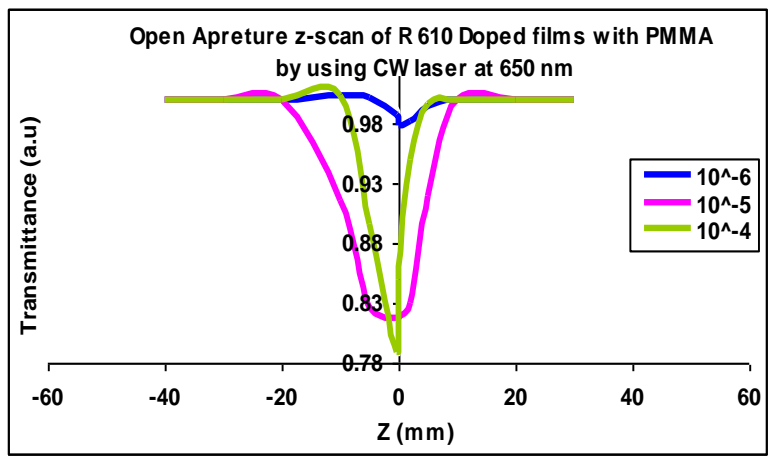

Fig.(6) OA Z-scan profile of R610 solid films of concentration $10^{-6} \mathrm{M}, 10^{-5} \mathrm{M}$, and $10^{-4} \mathrm{M}$ at $I_{0}=509.6 \mathrm{watt} / \mathrm{cm}^{2}$.

The linear transmittance is normalized to unity. When the sample is away from the focus $\mathrm{Z}=0$, the incident laser intensity is low and the normalized transmission is close to 1 ; when it moves close to the focus, the OA Z-scans exhibits a reduction in the transmission which is symmetric about the focus $\mathrm{Z}=0$ for all concentrations studied. This is typical of an intensity dependent enhanced absorption termed reverse saturable absorption (RSA) or positive nonlinear absorption exhibited by a nonlinear material [13], and can be exploited for protection of eyes and sensors against radiation induced damage.

Measurements of $\beta_{2}$ are performed using the following equation [8]:

The total transmittance is given by:

$T(z)=\sum_{m=0}^{\infty} \frac{\left[\frac{\beta I_{o} L_{\text {eff }}}{1+(Z / Z))^{2}}\right]^{m}}{(m+1)^{3 / 2}}$

Where,

$Z$ : - is the sample position at the minimum transmittance, $m$ : - integer. 
T (z):- the minimum transmittance. The two terms in the summation are generally sufficient to determine $\beta$.

$L_{\text {eff. }}$ : - the effective length of the sample, can be determined from the following formula: [11]

Leff $=\left[1-\exp \left(-\alpha_{0} L\right)\right] / \alpha_{0}$

Where, $\mathrm{L}$ is the sample length, $\alpha_{0}$ is the linear absorption coefficient,

$$
\alpha_{o}=\frac{1}{L \ln \left(\frac{1}{T}\right)}
$$

Where T: linear transmittance

$\mathrm{I}_{0}=2 \mathrm{P}_{\text {peak }} / \pi \omega_{0}$

where, $\omega_{0}$ : - the beam radius at the focal point, $\mathrm{P}_{\text {peak: - the peak power, }}$

$\mathrm{Z}_{0}=\mathrm{k} \omega_{0}^{2} / 2$

$\mathrm{Z}_{0}$ is the diffraction length of the beam, $\mathrm{k}=2 \pi / \lambda$, the wave number, and $\lambda$, the wavelength of the laser light [14].

For closed aperture z- scan we define the change in transmittance between the peak and valley in a Z-scan as:

$\Delta \mathrm{T}_{\mathrm{pv}}=\mathrm{T}_{\mathrm{p}}-\mathrm{T}_{\mathrm{V}}$

Where $T_{p}$ and $T_{v}$ are the normalized peak and valley transmittances as seen in Fig.(3). The empirically determined relation between the induced on axis phase shift, $\Delta \Phi_{0}$, and $\Delta \mathrm{T}_{\mathrm{pv}}$ for a third-order nonlinear refractive process in the absence of nonlinear absorption (NLA) is, [2]

$$
\left.\Delta T_{p \cong 0} \cong 0.40 \phi 1-S\right)^{0.27} \mid \Delta \phi_{0}
$$

S: the size of the aperture.

The size of the aperture is signified by its transmittance. In most reported experiments, $0.1<\mathrm{S}<0.5$ has been used for determining nonlinear refraction. $S$ the aperture linear transmittance is given by [13]:

$\mathrm{S}=1-\exp \left(-2 \mathrm{r}_{\mathrm{a}}^{2} / \omega^{2}{ }_{\mathrm{a}}\right)$

with $r_{a}$ denoting the aperture radius( it is $0.5 \mathrm{~mm}$ ), and, $\omega_{\mathrm{a}}$ denoting the radius of the laser spot before the aperture (it is $0.015 \mathrm{~mm}$ ). In our experiment $\mathrm{S}=1.12 \mathrm{E}-4$ then $(1-1.12 \mathrm{E}-4)$ ${ }^{0.27}=0.999999999 \approx 1$ ) so:
$\Delta T_{p v} \cong 0.40 \otimes \phi_{\mathrm{o}}$

and this analogy with Mansoor Sheik-Bahae, 2007[15].

The nonlinear refractive index is calculated from the peak to valley difference of the normalized transmittance by the following formula: - [6, 8 and 15]

$\mathrm{n}_{2}=\Delta \phi_{0} / \mathrm{I}_{\mathrm{o}} \mathrm{L}_{\mathrm{eff}} \mathrm{k}$

Closed aperture Z-scan profile (The defocusing effect) of R610 solid film at concentration $10^{-6}, 10^{-5}$ and $10^{-4} \mathrm{M}$ for $\mathrm{Io}=509.6 \mathrm{GW} / \mathrm{cm}^{2}$ are shown in Fig.(7).

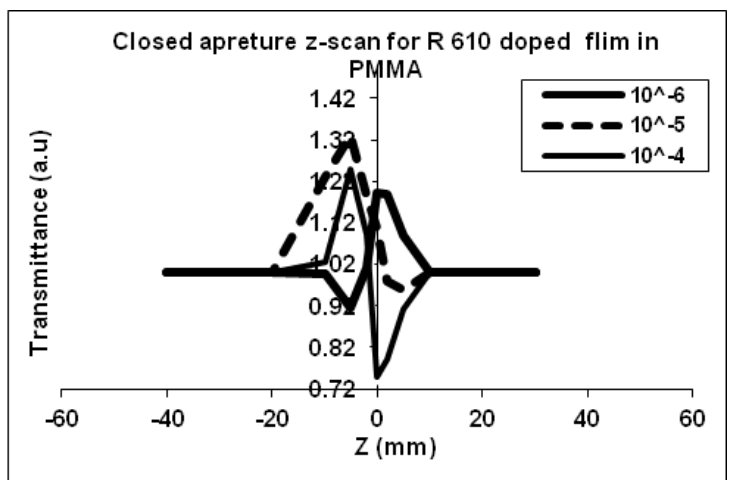

Fig. (7) CA Z-scan profile of R610 solid film at concentration $10^{-6}, 10^{-5}, 10^{-4} \mathrm{M}$ for $\mathrm{Io}=$ $509.6 \mathrm{GW} / \mathrm{cm}^{2}$ indicates to positive refractive index for film of concentration $\left(10^{-6}\right) \mathrm{M}$, and negative refractive index for films of concentration $\left(10^{-5}\right.$ and $\left.10^{-4}\right) M$.

Fig (7) indicates to positive refractive index for film of concentration $\left(10^{-6}\right) \mathrm{M}$, and negative refractive index for films of concentration $\left(10^{-5}\right.$ and $\left.10^{-4}\right) \mathrm{M}$ is attributed to a thermal nonlinearity resulting from the absorption of radiation at $650 \mathrm{~nm}$. Analogy to the theoretical behavior of the dotted line in Fig.(4).

Experimentally determined nonlinear refractive index $\mathrm{n}_{2}$ and nonlinear absorption coefficient $\beta$ can be used in finding the real and imaginary parts of the third-order nonlinear optical susceptibility $\left[\chi^{3}\right]$ according to the following: [15]

$\operatorname{Re} \chi^{(3)}(\mathrm{esu})=10^{-4} \varepsilon_{0} c^{2} n_{0}{ }^{2} n_{2} / \pi\left(\mathrm{cm}^{2} / \mathrm{W}\right)$ 
$\operatorname{Im} \chi^{(3)}(\mathrm{esu})=10^{-2} \varepsilon_{0} c^{2} n_{0}{ }^{2} \lambda \beta / 4 \pi^{2}(\mathrm{~cm} / \mathrm{W})$

Where $\varepsilon_{0}$ is the vacuum permittivity, and $c$ is the light velocity in vacuum. [16]

The absolute value of the third-order nonlinear optical susceptibility is given by the relation: $\left.\left.\mid \chi^{3} \|=\left[\left(\operatorname{Re} \chi^{3}\right)\right)^{2}+\left(\operatorname{Im} \chi^{3}\right)\right)^{2}\right]^{1 / 2}$

The experimentally determined values of $T \mathrm{p}-\mathrm{v}, \mathrm{n}_{0,}, n_{2}, \Delta$ opo and $\mathrm{R} \chi 3$ are given in Table (1). The experimentally determined values of $\beta, \operatorname{Im} \chi^{3}, \chi^{3}$. are given in Table (2).

Table (1)

Nonlinear parameters for PMMA Films Doped with R610 in Chloroform with various Concentrations of dye by using $\mathrm{CW}$ diode laser at $650 \mathrm{~nm}$ for closed aperture.

\begin{tabular}{|c||c||c||c|c|c|}
\hline $\boldsymbol{C}(\boldsymbol{M})$ & $\boldsymbol{T} \boldsymbol{p}-\boldsymbol{v}$ & $\boldsymbol{n}_{\boldsymbol{o}}$ & $\boldsymbol{n}_{2}\left[\mathbf{c m}^{2} / \boldsymbol{W}\right]$ & $\Delta$ qo & $\boldsymbol{R} \chi^{3}$ esu \\
\hline \hline $10^{-6}$ & 0.343 & 1.56 & $1.813 \mathrm{E}-06$ & 0.844 & $1.12 \mathrm{E}-02$ \\
\hline $10^{-5}$ & 0.290 & 1.55 & $3.067 \mathrm{E}-06$ & 0.715 & $1.88 \mathrm{E}-02$ \\
\hline $10^{-4}$ & 0.714 & 1.94 & $4.628 \mathrm{E}-06$ & 1.758 & $4.42 \mathrm{E}-02$ \\
\hline \hline
\end{tabular}

Table (2)

Nonlinear parameters for PMMA Films Doped with R610 in Chloroform with various Concentrations of dye by using $C W$ diode laser at $650 \mathrm{~nm}$ for open aperture.

\begin{tabular}{|c|c||c||c||c|}
\hline $\boldsymbol{C}(\boldsymbol{M})$ & $\boldsymbol{T}(z)$ & $\boldsymbol{\beta}(\mathbf{c m} /$ watt $)$ & $\operatorname{Im} \chi^{3}(\mathbf{e s u})$ & $\begin{array}{c}\chi 3 \\
(\text { esu })\end{array}$ \\
\hline \hline $10^{-6}$ & 0.289 & 0.405 & 1.639 & 1.280 \\
\hline $10^{-5}$ & 0.678 & 1.085 & 2.814 & 1.678 \\
\hline $10^{-4}$ & 0.844 & 1.305 & 3.413 & 1.848 \\
\hline
\end{tabular}

From Fig. $(8 \mathrm{a}$ and $b)$, there is an increasing trend for the values of $n_{2}$ and $\beta$ as the concentration increases. This may be attributed to the fact that the number of dye molecules increases as the concentration increases, more particles are thermally agitated resulting in an enhanced effect.
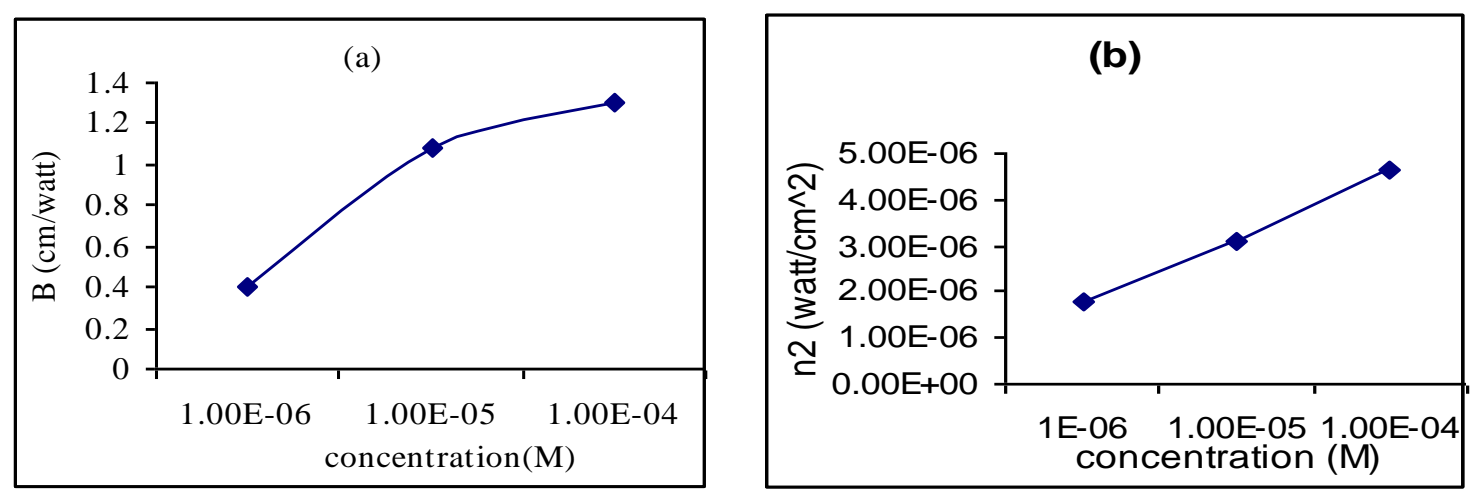

Fig. (8) Concentration dependence $n 2$ (a) and $\beta$ (b) of $R 610$ dye in polymer film.

With cw pumping we expect major contribution to the observed third-order nonlinearities to be thermal in nature. The energy from the focused laser beam is transferred to sample through linear absorption and is manifested in terms of heating the 
medium leading to a temperature gradient and there by the refractive index change across the sample which then acts as a lens. The phase of the propagating beam will be distorted due to the presence of this thermal lens. The peakvalley separation of more than 1.7 times the Rayleigh range of $\sim 0.0138 \mathrm{~cm}$ also suggests the presence of thermal component in our case. It is well established that a separation of $\sim 1.7 \mathrm{z}_{0}$ indicates Kerr-type of nonlinearity [1 and 16].

It is worth noting that the value of $\chi^{3}$ for the dye studied is larger than those of some representative third-order nonlinear optical materials such as safranin $O$ [1] dye and its derivatives and organic dyes like Mercurochrome [2].

\section{Conclusion}

The third order nonlinear optical properties of R 610 dye have been studied. Both NLA and NLR contribute to the large third-order nonlinearity of the dye. The origin of optical nonlinearity observed in the $\mathrm{cw}$ regime is attributed to the thermal variation of refractive index in the medium. The aperture limited designs based on thermo-optic nonlinearity such as the one studied here can be used as efficient limiters in the $\mathrm{CW}$ regime. There is an increasing trend for the values of $n_{2}$ and $\beta$ as the concentration increases. This may be attributed to the fact that the number of dye molecules increases as the concentration increases, more particles are thermally agitated resulting in an enhanced effect.

These samples will find potential applications in optical limiting and signal processing applications.

\section{Reference}

[1] G. Balaji, R.K. Rekha and A. Ramalingam," Nonlinear Characterization of Safranin O Dye for Application in Optical Limiting", Acta Physica Polonica A,Vol.119,No 3, 359363, 2011.

[2] Rekha Rathnagiri Krishnamurthy, Ramalingam Alkondan," Nonlinear characterization of Mercurochrome dye for potential application in optical limiting", Vol. XL, No. 1, pp187196,2010.
[3] Achamma Kurian, Nibu A George, Binoy Paul, V. P. N. Nampoori And C. P. G. Vallabhan," Studies on Fluorescence Efficiency and Photodegradation of Rhodamine 6G Doped PMMA Using a Dual Beam Thermal Lens Technique," Laser Chemistry, Vol. 20(2-4), pp. 99110, 2002.

[4] Przhonska O.V., Jin Hong Lim, Hagan D.J., Van Stryland E.W., Bondar M.V., Slominsky Yu.L., "Nonlinear light absorption of polymethine dyes in liquid and solid media", Journal of the Optical Society of America B, 15(2), pp. 802809, 1998,.

[5] Geethakrishnan T., Palanisamy P.K., "Zscan determination of the third-order optical nonlinearity of a triphenylmethane dye using $633 \mathrm{~nm}$ He-Ne laser" , Optics Communications 270(2), pp. 424-428, 2007.

[6] Mansoor Sheik -Bahae, A. A. Said, and E. W. Van Stryland, center of Research in Electro- Optics and Lasers, University of Florida, Orlando 1986.

[7] Uliich Brackmann, "Lambdacxhrome Laser Dyes", Lambdaphysics, Gm bH, 1986.

[8] Mansoor Sheik- Bahae, Michael P Hassaibeak," 2000.

[9] M. Sheik- Handbook of Optics, Vo.IV, Chapter 17,(Bahae, A. Said, T.H Wei, D.J Hagan, E.W Stryland, IEEE Journal of Quantum electronics. 26, 760-769 1990.

[10] K. Kandasamy, K. Divakar Rao, Rekha Deshpande, P.N Puntambekar, B.P Singh, S.J Shetty, T.S Srivastava, Appl. Phys.B 64, 479-484, 1997.

[11] P.G Louie Frobel, R.Sreeja, C.IMuneera, and M.K Jayaraj, "Nonlinear Optical Characterization of Biebrich Scarlet dyedoped PVA solid films under $532 \mathrm{~nm}$ nanopulsed laser light excitation", ICOP 2009-International Conference on Optics and Photonics CSIO,Chandigarh,India,30 Oct.-1 Nov.2009.

[12] G. Cerullo, S. De Silvestri, and V. Magni, "Self-starting Kerr-lens mode locking of a Ti:sapphire laser", Optics Letters, Vol. 19, pp. 1040-1042, 1994. 
[13] R.K. Rekha and A. Ramalingam, "Nonlinear characterization and optical limiting effect of carmine dye", Indian Journal of Science and Technology, Vol.2, No. 8,pp 27-31, Aug 2009.

[14] F_elix Fern_andez-Alonso a, Paolo Marovino b, Anna Maria Paoletti b, Marcofabio Righini a, Gentilina Rossi b," Third-order optical non-linearities in titaniumbis-phthalocyanine/toluene solutions", Chemical Physics Letters, Vol. 356, pp 607-613, 2002.

[15] Mansoor Sheik-Bahae,, David J. Hagan,, "Sensitive Measurement of Optical Nonlinearities, Using a Single Beam", IEEE LEOS NEWSLETTER. Pp17-26, February 2007.

[16] S.J Mathews, S. Chaitanya Kumar, L.Giribabu, S. Venugopal Rao, "Nonlinear optical and optical limiting properties of phthalocyanines in solution and thin films of PMMA at $633 \mathrm{~nm}$ studied using a cw laser", Materials Lett. Vol. 61, 4426-4431, 2007.

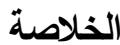

نقدم في هذه الورقة البحثية دراسة للصفات اللاخطية

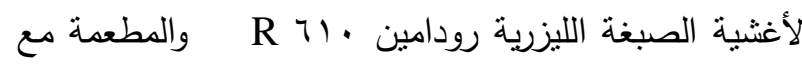

البوليمر (PMMA) بتراكيز مختلفة بأستخدام المذيب

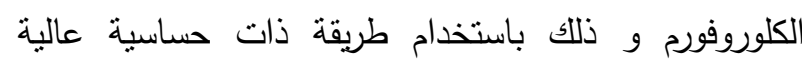

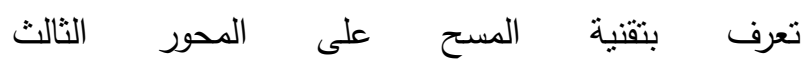

Z-scan

باستخدام ليزر الدايود ذو الموجات المستمرة عند طول موجي

. 7 نانومتر و قدرة خارجة 0؛ ملي وات على جزئيين:

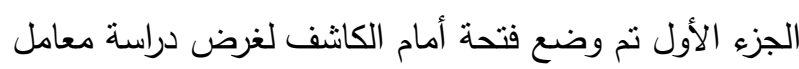

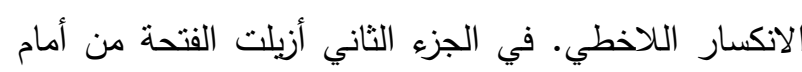

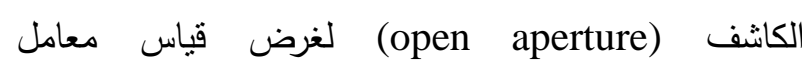

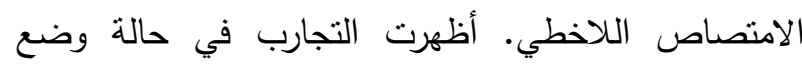

الفتحة أمام الكاثف إن أغشية الصبغة المطعمة تظهر معامل

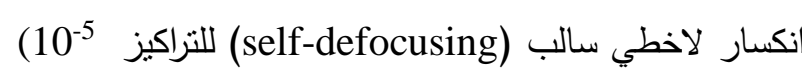

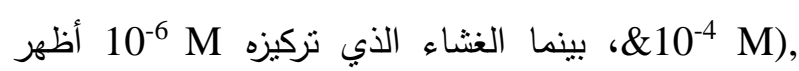

إنكسار موجب (self-focusing).أظهرت التجارب ايضا ان

معامل الإنكسار اللاخطي وقابلية التأثراللاخطية من الدرجة

الثالثة (third order susceptibility) تزداد بزيادة التركيز
وذللك لزيادة سمك الغشاء الذي يؤدي الى زيادة إزاحة

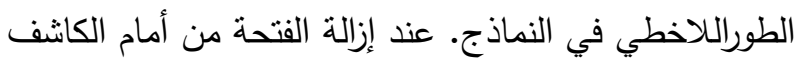

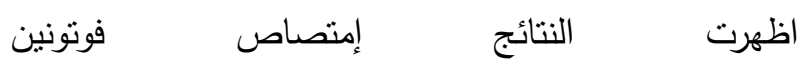
. Two photon absorption R610 بإمكانها أن تكون أوساط لمختلف التطبيقات الكهربائية الضوئية تشتمل على المحددات البصرية للقدرة. 